\title{
EPR FINGERPRINTING AND ANTIOXIDANT RESPONSE OF FOUR SELECTED PLANTAGO SPECIES
}

\author{
ANCA D. FARCAȘ ${ }^{\mathrm{a}, \mathrm{b}}$, CEZARA ZĂGREAN-TUZAc, LAURIAN VLASE $^{\mathrm{d}}$, \\ ANA-MARIA GHELDIUd, MARCEL PÂRVUª , AUGUSTIN C. MOṬ",b*
}

\begin{abstract}
In this study we compare the phytochemical composition and antioxidant properties of four Plantago species ( $P$. cornuti, $P$. lanceolata, $P$. major and $P$. media), in terms of radical scavenging ability and their reductive properties in corroboration with EPR spectroscopy profiling. We found that Plantago extracts possess very low prooxidant activity while being efficient radical scavengers and electron donors. $P$. media showed the strongest antioxidant activity from the four species investigated. The relative composition derived from the EPR spectra of the studied extracts was compared to the phytochemical composition determined by HPLC-MS analysis. Downhill Simplex algorithm simulation of EPR experimental signals revealed that all four Plantago extracts display luteolin and chlorogenic acid profiles, as well as quercetin glycosides, especially rutin. EPR spectroscopy appears to be a useful tool for discrimination of radical-based complex mixture, rendering it as an alternative method for predicting chemical behaviour, regardless of synergistic or antagonistic effects.
\end{abstract}

Keywords: Plantago, Antioxidants, EPR fingerprinting

\section{INTRODUCTION}

A genus comprising more than 200 species, Plantago herbs are known throughout the world as medicinal plants ${ }^{1}$. Known for their effects since Antiquity, aerial parts of these plants are still used as natural remedies

\footnotetext{
a Babeş-Bolyai University, Faculty of Biology and Geology, Cluj-Napoca 400028, Romania

${ }^{b}$ National Institute for Research and Development of Isotopic and Molecular Technologies, Cluj-Napoca 400293, Romania

c Babeş-Bolyai University, Faculty of Chemistry and Chemical Engineering, Cluj-Napoca 400028, Romania

d Iuliu Hațieganu University of Pharmacy and Medicine, Faculty of Pharmacy, Cluj-Napoca 400012, Romania

*Corresponding author: augustinmot@chem.ubbcluj.ro
} 
for several diseases, including inflammation, skin, respiratory, digestive and reproductive organs issues, as well as cancer [1-4]. Nowadays, there is an upsurge of interest in the therapeutic potential of medicinal plants, mostly because of their effectiveness in prevention, as well as for their low toxicity, which triggers less side effects as compared to the synthetic conventional remedies. In many countries, Plantago herbs, especially $P$. lanceolata, are also appreciated for their nutritional values in both humans and animals [5], [6].

The most renowned Plantago species still used in modern medicine are $P$. major, $P$. lanceolata, $P$. media, $P$. indica, $P$. ovata, $P$. asiatica and $P$. sempervirens [11]. Among others, a widely used therapeutic perspective is the antioxidant nature of the medication which helps in prevention, neutralization of harmful reactive species and maintainance of a normal redox balance of the cells. The antioxidants are chemical compounds known to have the ability of scavenging reactive oxygen or nitrogen species [12]. Many Plantago species have been investigated for their antioxidant capacity $[2,3,6,13]$ by employing various assays, both in vitro and in vivo. Thereby, there is a particular interest in the study of new sources of natural antioxidants, which have less side effects compared to classic drugs and could be used to prevent and to treat a large spectrum of disorders.

We have explored in a previous study [14] the phytochemical composition, antioxidant and anti-inflammatory effects of $P$. sempervirens Crantz. Following this, we decided to survey the phytochemical potential of other Plantago species mentioned above. Therefore, our paper aims at making a general in vitro investigation of four Plantago species (P. cornuti, P. lanceolata, $P$. major and $P$. media), determining their antioxidant and pro-oxidant activity and highlighting their major phytochemical constituents, with a strong focus on an EPR spectroscopy method, which enables a degree of fast fingerprinting.

\section{RESULTS AND DISCUSSION}

Polyphenolic profile of the four Plantago samples ( $P$. cornuti, $P$. lanceolata, $P$. major and $P$. media) follows a common composition, but each extract distinguishes itself by different amounts of polyphenols (Table 1). The basic phytochemical profile for all four species was made from luteolin and apigenin; regarding luteolin, by far $P$. lanceolata extract holds the highest amount as compared to the other Plantago counterparts. The highest concentration of chlorogenic acid was found in $P$. lanceolata and $P$. media. 
Increasing the plant extracts $\mathrm{pH}$ with basic reagents like sodium hydroxide gives rise to EPR signals due to oxidation of secondary metabolites, mainly polyphenols, in the presence of molecular oxygen. In this way, flavonoids and phenolic acids are prone to transformation into reactive anionic radicals exhibiting specific EPR features due to their distinct hyperfine coupling of protons in the vicinity of the unpaired electron. However, not every phytoconstituent can be converted into an anionic radical; in fact, there are some structural features that give rise to a relatively stable intermediate such as extensive conjugation, presence of a phenyl substituent as the center for radical formation and hydroxyl groups in key position [15]. Also, the reactivity of these intermediates varies greatly, thus affecting their half-life.

Table 1. The polyphenolic composition in the studied species (ug/g plant material)

\begin{tabular}{|l|l|l|l|l|}
\hline $\begin{array}{l}\text { Polyphenolic } \\
\text { compounds }\end{array}$ & $\boldsymbol{P}$. cornuti & $\boldsymbol{P}$. lanceolata & $\boldsymbol{P}$. major & $\boldsymbol{P}$. media \\
\hline Gentisic acid & $<0.2$ & $<0.2$ & $<0.2$ & $<0.2$ \\
\hline Caffeic acid & $<0.2$ & $<0.2$ & $<0.2$ & $<0.2$ \\
\hline $\begin{array}{l}\text { Chlorogenic } \\
\text { acid }\end{array}$ & $<0.2$ & 8.253 & $<0.2$ & 1.836 \\
\hline $\begin{array}{l}\boldsymbol{p} \text {-cumaric } \\
\text { acid }\end{array}$ & $<0.2$ & $<0.2$ & $<0.2$ & 0.501 \\
\hline Ferulic acid & $<0.2$ & $<0.2$ & $<0.2$ & $<0.2$ \\
\hline Rutin & $<0.2$ & 8.253 & $<0.2$ & $<0.2$ \\
\hline Luteolin & 6.487 & 23.214 & 1.021 & 0.951 \\
\hline Apigenin & 0.383 & 0.677 & 1.755 & 0.285 \\
\hline Quercitrin & $<0.2$ & $<0.2$ & $<0.2$ & $<0.2$ \\
\hline
\end{tabular}

The EPR profiles of the studied extracts at four different concentrations are presented in Figure 1. The EPR spectrum at the highest dilution, corresponding to each Plantago extract was simulated using HPLC identified components: luteolin, chlorogenic acid and an rutin. Apigenin exhibits no EPR spectrum in our conditions, indicating a higher redox potential or a low stability of the generated radicals in basic medium. 
A.

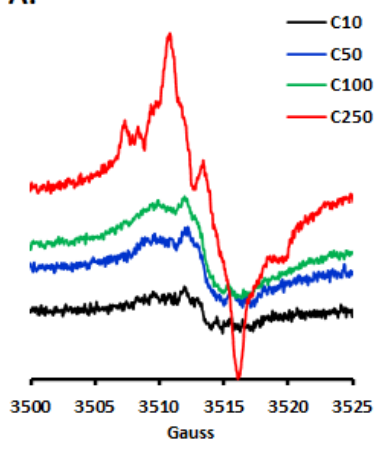

D.

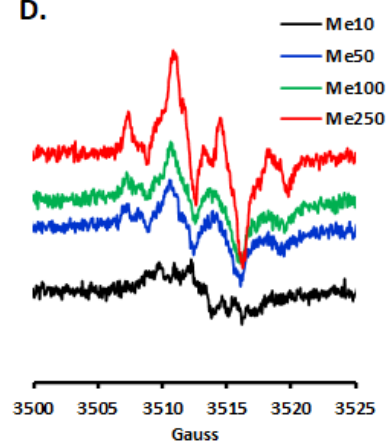

B.
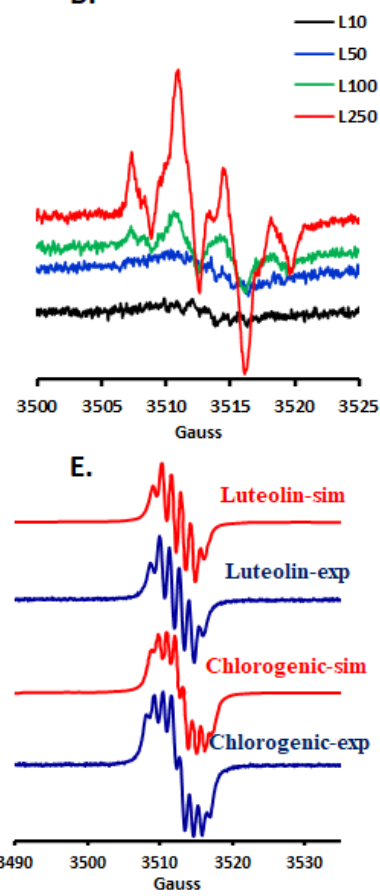

C.
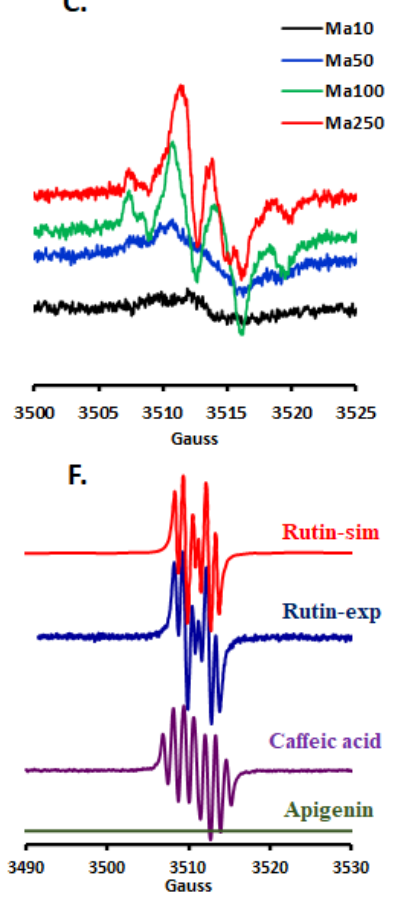

Figure 1. EPR spectra profiles for the four extracts of Plantago. (A) P. cornuti, (B) P. lanceolata, (C) P. major, (D) P. media, using different dilutions in $75 \%$ ethanol (10x, 50x, 100x and 250x times). EPR spectra of luteolin, chlorogenic acid and rutin, experimental and simulated and experimental EPR spectra of caffeic acid and apigenin, measured in the same conditions as extracts (E, F).

The entry parameters for all three components were those previously optimized for the pure standard; for each signal, optimization occurred via Downhill Simplex algorithm, thus determining the relative contribution for each component. The results are presented in Table 2; the simulated and their corresponding experimental spectra are depicted in Figure 2. All simulations exhibited correlation coefficients with experimental signal higher than 0.98 .

Table 2. EPR signal contribution for main phytochemical components in the four Plantago extracts

\begin{tabular}{|l|l|l|l|l|}
\hline Simulated EPR signal & $\boldsymbol{P}$. cornuti & $\boldsymbol{P}$. lanceolata & $\boldsymbol{P}$. major & $\boldsymbol{P}$. media \\
\hline Luteolin & $53.9 \%$ & $37.2 \%$ & $33.2 \%$ & $50.8 \%$ \\
\hline Chlorogenic acid & $22.9 \%$ & $26.3 \%$ & $21.7 \%$ & $31.9 \%$ \\
\hline Rutin & $23.2 \%$ & $36.5 \%$ & $45.1 \%$ & $17.3 \%$ \\
\hline
\end{tabular}



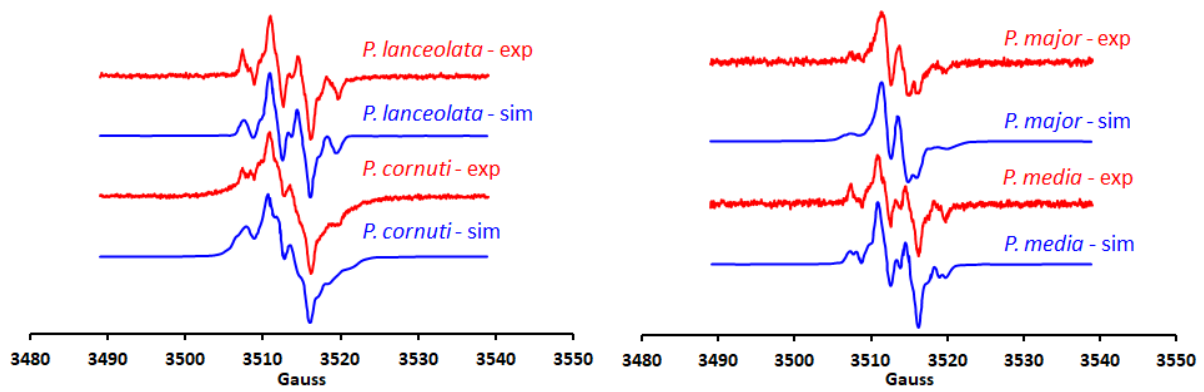

Figure 2. Experimental (exp) and simulated (sim) EPR spectra profiles for the four studied extracts of Plantago.

Each of standards determined using HPLC analysis was simulated separately, considering possible hyperfine couplings with neighbouring protons: luteolin radical lone electron interacts with four different protons, whereas chlorogenic acid possesses five distinct protons that are coupled with the single electron. The third EPR standard considered was rutin, as HPLC results pointed out. At a glance, it is obvious that the width and signal shape is influenced by chlorogenic acid, whilst the hyperfine splitting are due to luteolin and rutin.

The EPR contribution for each component was in accord with the HPLC results. Regardless of HPLC detected concentration magnitude in each extract, luteolin displayed a notable contribution in the EPR spectrum. It is interesting to note that $P$. lanceolata did not exhibit the highest luteolin simulated contribution, despite the fact that HPLC analysis pointed out to the highest concentration of this flavonoid; a possible explanation for this observation can be the existence of antagonistic effects that arise due to the phytochemical complexity of the extract, which can alter the signal shape as well as the semiquinone radical half-life. When it comes to chlorogenic acid, the second simulated standard, all extracts display a relatively similar EPR contribution, mostly according to HPLC results. Interestingly, $P$. cornuti and $P$. major do not exhibit detectable levels of this phenolic acid in HPLC. This can be explained by the fact that some compounds have strong EPR fingerprints that can be seen even in much lower concentrations. As for rutin, the variation in area value is great. $P$. major followed by $P$. lanceolata have the strongest resemblance to rutin signal shape; indeed, HPLC analysis pointed to detectable levels of rutin in the latter. Still, the third component of the simulated signals might be due not only rutin, but also to other quercetin derivatives like quercitrin and isoquercitrin. Indeed, all four signal are mainly similar in appearance to luteolin and chlorogenic acid spectra, whereas additional hyperfine features may arise from rutin and other quercetin glycosides. 
The antioxidant properties of polyphenolic compounds still represent a topic of scientific interest, due to phytoconstituents potential as alternative treatments or food supplements [16]. A first line of assessments used to test the protective potential of ethanolic extracts was made in terms of in vitro antioxidant activity. Artificial free radicals such as DPPH and ABTS can be used as targets for antioxidant routine tests, whereas Folin-Ciocâlteu method quantifies the total phenolic content, indirectly related to free radical quenching ability. Figure 3 (A, B and C) depicts a comparative antioxidant analysis of the four tested extracts; $P$. media showed a significant higher antioxidant activity as compared to its Plantago counterparts.

A.

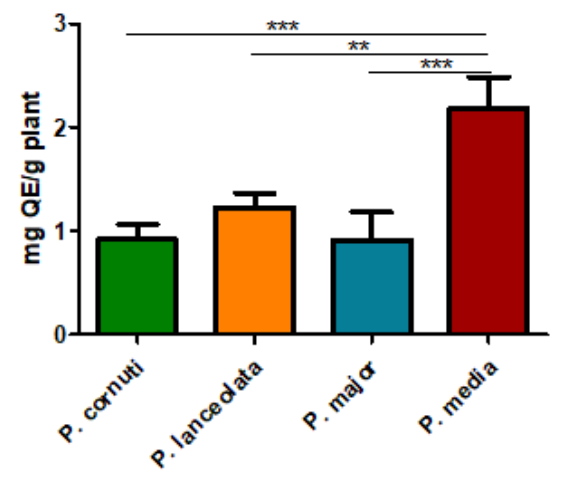

C.

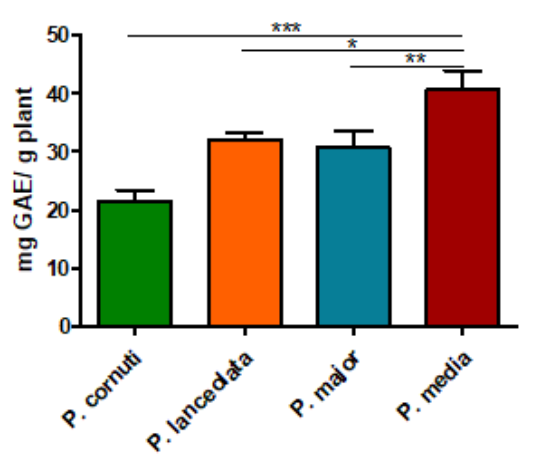

A.

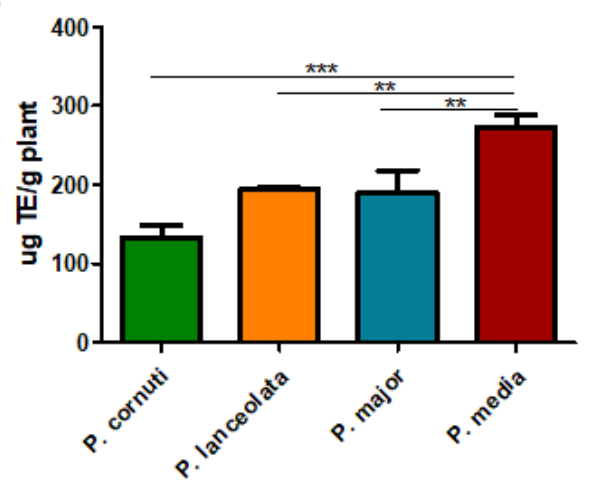

D.

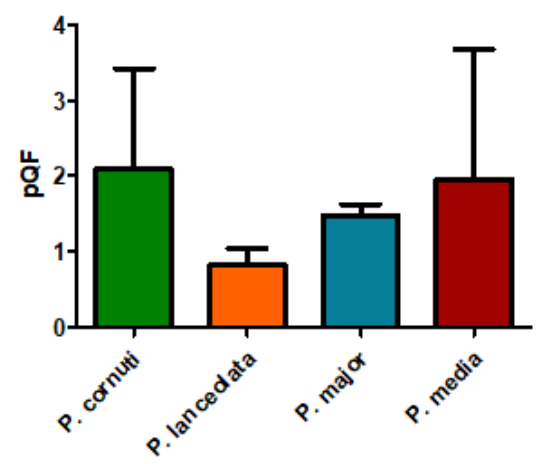

Figure 3. Antioxidant activity of the four studied extracts of Plantago as determined using DPPH bleaching assay (A), ABTS scavenging method (B), total phenolic content using Folin-Ciocâlteau assay $(C)$ and the pro-oxidant enzymatic method (D). Statistical comparison using ANOVA test: ${ }^{* *}$ Significant at $p<0.001$ as compared to $P$. cornuti, $P$. lanceolata, $P$. major and $P$. media, ${ }^{\text {S }}$ Significant at $\mathrm{p}<0.01$ as compared to $P$. cornuti. Values are the mean $\pm \operatorname{SD}(n=3)$. 
The TEAC results correlate well with the DPPH values; $P$. media once again ranks first $(p<0.001)$ as compared to its Plantago counterparts. Regarding Folin-Ciocâlteu (FC) assay, $P$. media expressed significant reduction ability $(40.65 \pm 1.85 \mathrm{mg} \mathrm{GAE} / \mathrm{g}, \mathrm{p}<0.01)$ as opposed to $P$. cornuti, $P$. lanceolata and $P$. major. Therefore, three different assays designate $P$. media as displaying the strongest antioxidant potential within the four species we have investigated. None of the four hydroalcoholic extracts exhibited significant pro-oxidant activity, as seen in Figure 3D. Such information might lead to further investigations regarding the beneficial effects of the Plantago extracts.

Many herbs have been found to possess antioxidant properties, which should therefore minimise redox imbalances or counteract the effects of free radicals. It is well known that antioxidants could scavenge reactive species (ROS/NOS) [5]. A strong antioxidant activity was yielded by $P$. media against DPPH and ABTS radicals, whereas use of Folin-Ciocâlteu reagent underlined a high concentration of reductive species. These results are in a reasonable agreement with the quantitative chromatographic analysis, due to higher amounts of polyphenols found in this extract. There have been many papers regarding Plantago species, starting with phytochemical analysis and other biological activities [5,8,13,17-22]. In addition, the antioxidant function derived from in vitro assays confirms the high potential of $P$. media extract and supports its further use in more complex tests. There were considerable variations between the samples from the perspective of antioxidant and chromatographic methods employed in this preliminary work. Previous studies discussed the antioxidant activity and polyphenolic content in $P$. lanceolata, $P$. cornuti and $P$. major $[3,4,6,12,23,24]$, without $P$. media. It is noteworthy that there are a few remarks concerning $P$. major and its antioxidant potential which is not exactly proper for therapeutic purposes since the free radical generation does not resemble the physiological conditions [23]. Depending on the $\mathrm{pH}$ of the environment, dose and enzymatic interactions, polyphenolic compounds can behave as antioxidants or can display prooxidant properties $[26,30]$. Therefore, a strong antioxidant activity can operate in two different ways: antioxidant or pro-oxidant. Considering this, antioxidant/prooxidant balance represents a key factor for an efficient natural therapy, which could be elucidated by continuing research in a multiple dimension, such as in vitro or in vivo studies. Studies conducted on model biosystems, such as laboratory animals, offer the possibility of investigating the antioxidant/prooxidant properties of plant extracts rich in polyphenolic compounds and of tracking the physiological effects in various experimental patterns of oxidative stress. 
ANCA D. FARCAȘ, CEZARA ZĂGREAN-TUZA, LAURIAN VLASE, ANA-MARIA GHELDIU, MARCEL PÂRVU, AUGUSTIN C. MOȚ

\section{CONCLUSIONS}

The antioxidant potential and the pro-oxidant reactivity of the four Plantago extracts (P.cornuti, P. lanceolata, P.major and P. media) were investigated using radical scavenging assay and, hemoglobin-oxidation, respectively in presence of laccase. No significant pro-oxidant activity was found by this enzymatic assay. Follow up the EPR fingerprintion in alkali medium, all Plantago extracts are dominated by luteolin and chlorogenic acid, as well as rutin. The signals might comprise other phytochemical fingerprints that, due to smaller concentration or shorter half-life, could have not been detected through this method. Sinergistic and antagonistic effects should be taken into consideration as far as they can give rise to higher reactivity and, as a result, a shorter radical lifespan. Thus, EPR spectroscopy in combination with a simulating tool software such as WinSim can offer a sneak peek into plant extracts relative composition. The highest antioxidant activity was attributed to $P$. media extract, thus nominating it as a strong candidate for further in vitro and in vivo tests.

\section{EXPERIMENTAL SECTION}

\section{Plant material and extracts preparation}

The leaves of $P$. cornuti, $P$. lanceolata, $P$. major and $P$. media were collected from the Botanical Garden "Alexandru Borza" from Cluj-Napoca, Romania in September 2015 and were identified at the Herbarium of the Botanical Garden, where a voucher specimen was deposited for each plant (P. cornuti- CL663371, P. lanceolata- CL663372, P. major- CL663369, P. media- CL663370). The Plantago extracts were prepared by the percolation method at room temperature for three days by using small fragments $(0.5-1$ $\mathrm{cm}$ ) of fresh leaves and $70 \%$ ethanol. The fluid extract of Plantago contained $35 \%$ ethanol and was: $1: 1.4$ for $P$. cornuti; $1: 1.7$ for $P$. lanceolata; $1: 1.3$ for $P$. major and $P$. media [27].

\section{Chemicals}

Folin-Ciocâlteu reagent, DPPH (di(phenyl)-(2,4,6-trinitrophenyl) iminoazanium), ABTS (2,2'-azino-bis(3-ethylbenzothiazoline-6-sulfonic acid)), $\mathrm{N}$-(1-naphtyl)ethilenediaminedihidrochloride, sulphuric acid, hydrochloride acid, glycerol, trolox (6-hydroxy-2,5,7,8-tetrametyl-croman-2-carboxylic acid) were obtained from several companies (Sigma, Fluka, Merck). Standards: rutin, kaempferol, chlorogenic acid were purchased from Sigma, Germany. Ferulic acid, galic acid, luteolin and quercetin were obtained from Roth, Germany. All other chemicals and solvents used in the study were of analytical grade. 


\section{HPLC-MS analysis}

The phytochemical analysis was carried out using an Agilent Technologies 1100 HPLC Series system (Agilent, Santa Clara, CA, USA) assisted by mass spectrometry, an Agilent 1100 Ion Trap SL (LC/MSD Ion Trap VL) equipped with an electrospray or ACPI ion source. For the separation, a reverse-phase analytical column was employed (Zorbax SB-C18 100x3.0 $\mathrm{mm}$ i.d., $3.5 \mu \mathrm{M}$ particle). All chromatographic operations were carried out at $48^{\circ} \mathrm{C}$. The compounds were detected with both UV $(330 \mathrm{~nm})$ source and in MS mode (electrospray ion source in negative mode). The chromatographic data were processed using a software from Agilent, ChemStation and DataAnalysis. The mobile phase was made up of methanol and acetic acid $0,1 \%(v / v)$. The flow rate was $1 \mathrm{~mL} / \mathrm{min}$ and injection volume was $5 \mu \mathrm{L}$. Based on the chromatographic conditions described above and preliminary experiments, the compounds eluted in less than 40 minutes. There were a few compounds that were not quantified due to overlapping (gentisic acid and caffeic acid), but they were identified using MS detection. The limit of quantification was around $0.5 \mu \mathrm{g} / \mathrm{mL}$. We also performed quantitative determination, using external standard method. The calibration curves (0.5$50 \mu \mathrm{g} / \mathrm{mL}$ ) range with $R^{2}>0.999$ and detection limits between $17-90 \mathrm{ng} / \mathrm{mL}$. All reagents were of analytical grade purity.

\section{EPR Measurements}

The protocol for the Electron Paramagnetic Resonance (EPR)-based investigation is fully described elsewhere [28]. All measurements have been performed using an ELEXSYS $580 \mathrm{X}$ - band EPR spectrometer. The simulation of EPR spectra has been carried using WinSim 2002 software [29] on the signal with the highest resolution from all four dilutions, namely diluted 250 times. The standards chosen for comparison are luteolin and chlorogenic acid, along with rutin. Each EPR signal of the employed standards was simulated separately, then optimized by use of Downhill Simplex algorithm.

\section{Pro-oxidant activity assays}

The prooxidant reactivity of the extract was evaluated using a previously developed method that is described in detail elsewhere [30]. Briefly, the extract is treated with catalytic amount of laccase that generates radicals from the components of the extract which are responsible of oxidation of the ferrous oxy hemoglobin $(\mathrm{oxyHb})$ into the oxidized form $(\mathrm{metHb})$ which is unable to transport molecular oxygen. The kinetic profile and the rate of the oxyHb oxidation is a marker for the reactivity of the generated radicals and it is expressed as relative quercetin prooxidant factor using a quercetin standard (pQF). 
ANCA D. FARCAȘ, CEZARA ZĂGREAN-TUZA, LAURIAN VLASE, ANA-MARIA GHELDIU, MARCEL PÂRVU, AUGUSTIN C. MOȚ

\section{Antioxidant activity assays and total phenolic determination DPPH bleaching assay}

The DPPH solution $(900 \mu \mathrm{M})$ was prepared in ethanol and $111 \mu \mathrm{L}$ was added alongside with $50 \mu \mathrm{L}$ of Plantago extract. The final volume $(1 \mathrm{~mL})$ was adjusted with a solution made of ethanol and water (1:1). The bleaching of DPPH was examined after 40 minutes incubation at $517 \mathrm{~nm}$, using a UVvis spectrophotometer (Varian, Cary 50) and expressed in quercetin equivalents using a quercetin-based calibration curve $(n=6, R=0.999)$ [31]. The measurements were carried out in triplicates.

\section{Trolox equivalent antioxidant capacity (TEAC) assay}

In a quartz cuvette, $100 \mu \mathrm{L}$ of ABTS radical were added along with $10 \mathrm{mM}$ sodium acetate buffer $\mathrm{pH} 5.5$ and $50 \mu \mathrm{L} 10$ times diluted Plantago sample. The final volume was $1 \mathrm{~mL}$. The decreasing ABTS absorbance was monitored for 15 minutes at $420 \mathrm{~nm}$, using a UV-vis spectrophotometer (Varian, Cary 50). The measurements were done in triplicates. The results were expressed as trolox equivalent (TE) via a calibration curve $(n=6$, $\mathrm{R}=0.999$ ) using trolox standard solutions.

\section{Determination of total polyphenols content (GAE) of the extract}

Total polyphenols content of the Plantago extracts was determined using Folin-Ciocâlteu method [28]. The extract was diluted 10 times and 10 $\mu \mathrm{L}$ diluted solution was mixed with $790 \mu \mathrm{L}$ of ultrapure water, $50 \mu \mathrm{L}$ of FolinCiocalteu reagent and $150 \mu \mathrm{L}$ of $20 \%$ sodium carbonate. The final concentration of the plant extract in the assayed solution was $0.01 \mathrm{mg} / \mathrm{mL}$. The measurements were done in triplicates, both for the samples and for the standards. Gallic acid $(21 \mathrm{mg} / \mathrm{mL})$ was used as standard stock solution for calibration curve. Standards from 0.5 to $30 \mathrm{mg} / \mathrm{L}$ were prepared and treated along with the samples, then incubated for 30 minutes in the dark. The solutions turned blue and the absorbance was recorded at $750 \mathrm{~nm}$, using a UV-vis spectrophotometer (Varian, Cary 50). For each sample, the level of phenolics were determined in terms of gallic acid equivalents (GAE).

\section{Statistical analysis}

All data are reported as the mean \pm SD. The Gaussian distribution was checked by the Shapiro-Wilk normality test. One-way analysis of variance ANOVA, followed by Bonferroni's Multiple Comparison test procedure was performed. Statistical significance was at $p<0.05$. Statistical values were obtained using Statistica 12. 
EPR FINGERPRINTING AND ANTIOXIDANT RESPONSE OF FOUR SELECTED

PLANTAGO SPECIES

\section{ACKNOWLEDGMENTS}

Financial support from the National Authority for Scientific Research and Innovation - ANCSI, Core Programme, Project PN19 350201 is gratefully acknowledged.

The authors have no conflict of interest to declare.

\section{REFERENCES}

1. A.B. Samuelsen; J. Ethnopharmacol., 2000, 71,1-21.

2. M. Nikolova; L. Evstatieva; D. Nguyen; Garden, 2011, 35, 43-48.

3. T. Janković; G. Zdunic; I. Beara; K. Balog; D. Pljevljakusic; D. Stesevic; K. Savikin; Biochem. Syst. Ecol., 2012, 42, 69-74.

4. I. Stanisavljevic; S. Stojicevic; D. Velickovic; M. Lazic; V. Veljkovic; Sep. Sci. Technol., 2008, 43, 3652-3662.

5. S. Gonçalves; A. Romano; Ind. Crops Prod., 2016, 83, 213-226.

6. M. Gálvez; C. Martín-Cordero; P.J. Houghton; M.J. Ayuso; J. Agric. Food Chem., 2005, 53, 1927-1933.

7. N. Ronsted; E. Gobel; H. Franzyk; S.R. Jensen; C.E. Olsen; Phytochemistry, 2000, 55, 337-348.

8. M. Zubair; A. Ekholm; H. Nybom; S. Renvert; C. Widen; K. Rumpunen; J. Ethnopharmacol., 2012, 141, 825-830.

9. P. Lukova; I. Dimitrova-Dyulgerova; D. Karcheva-Bahchevanska; R. Mladenov; I. Iliev; M. Nikolova; Int. J. Med. Res. Pharm. Sci., 2017, 4, 20-26.

10. W. Reardon; D.A. Fitzpatrick; M.A. Fares; J.M. Nugent; Plant Mol. Biol., 2009, $71,241-250$.

11. I.N. Beara; M.M. Lesjak; E.D. Jovin; K.J. Balog; G.T. Anackov; D.Z. Orcic; N.M. Mimica-Dukic; J. Agric. Food Chem., 2009, 57, 9268-9273.

12. F. Pourmorad, S.J. Hosseinimehr; N. Shahabimajd; African J. Biotechnol., 2006, 5, 1142-1145.

13. I.N. Beara; M.M. Lesjak; D.Z. Orcic; N.D. Simin; D.D. Cetojevic-Simin; B.N. Bozin; N.M. Mimica-Dukic; LWT - Food Sci. Technol., 2012, 47, 64-70.

14. A. D. Farcaș; A.C. Moț; A.E. Pârvu; V.A. Toma; M.A. Popa; M.C. Mihai; B. Sevastre; I. Roman; L. Vlase; M. Pârvu; Oxid. Med. Cell. Longev. 2019, 2019, $1-13$.

15. S. Fiorucci; J. Golebiowski; D. Cabrol-Bass; S. Antonczak; J. Agric. Food. Chem., 2007, 55(3), 903.

16. J. B. Silva; R.F. Mendes; V. Tomasco; N.C.C. Pinto; L.G. deOliveira; M.N. Rodrigues; D.M.O. Aragao; J.A.K. Aguiar; M.S. Alves; M.C.N.M. Castagnon; A. Ribeiro; E. Scio; J. Ethnopharmacol., 2017, 198, 399-406.

17. A. Venditti; Nat. Prod. Res., 2011, 26(21), 2035-2039. 
ANCA D. FARCAȘ, CEZARA ZĂGREAN-TUZA, LAURIAN VLASE, ANA-MARIA GHELDIU, MARCEL PÂRVU, AUGUSTIN C. MOȚ

18. E. Reina; N. Al-Shibani; E. Allam; K.S. Gregson; M. Kowolik; L.J. Windsor; J. Tradit. Complement. Med., 2013, 3, 268-272.

19. E.F. Al-Jumaily; H.A. Abdul-Ratha; R.H. Raheema; DAMA Int., 2012, 1, 17-21.

20. H. Ravn; L. Brimer; Phytochemistry, 1988, 27, 3433-3437.

21. H. Fleer; E.J. Verspohl; Phytomedicine, 2007, 14, 409-415.

22. J.L. Hu; S.P. Nie; F.F. Min; M.Y. Xie; J. Agric. Food Chem., 2012, 60, $11525-$ 11532.

23. J.C. Mello; M.V.D. Gonzalez; V.W.R. Moraes; T. Prieto; O.R. Nascimento; T. Rodrigues; Molecules, 2015, 20(10),17747-17759.

24. R. Taskova; N. Handjieva; L. Evstatieva; S. Popov; Phytochemistry, 1992, 52, 1443-1445.

25. A.D. Farcaș; A.C. Moț; C. Zăgrean-Tuza; V.A. Toma; C. Cimpoiu; A. Hosu; M. Pârvu; I. Roman; R. Silaghi-Dumitrescu; PLoS ONE, 2018, 13(7):e0200022.

26. D. Procházková; I. Boušová; N. Wilhelmová; Fitoterapia, 2011, 82, 513-23

27. M. Pârvu; O. Roșca-Casian; M. Pușcaș, G. Groza; Contribuții bot., 2009, 44, 125-129.

28. A.C. Moț; G. Damian; C. Sârbu; R. Silaghi-Dumitrescu; Redox. Rep., 2009, $14,267-74$.

29. D.R. Duling, J. Magn. Reson. B; 1994, 104, 105-10.

30. A.C. Moţ; C. Coman; C. Miron; G. Damian, C. Sârbu, R. Silaghi-Dumitrescu; Food Chem., 2014, 143, 214-22.

31. A. Karadag; B. Ozcelik; S. Saner; Food Anal. Methods, 2009, 2, 41-60. 